\title{
Flexural behaviour of ABS 3D printed parts on professional printer Stratasys Fortus $900 \mathrm{mc}$
}

\author{
Pavel Stoklasek ${ }^{1, *}$, Milan Navratil ${ }^{1}$, Martin Bednarik ${ }^{1}$, Ivan $\mathrm{Hudec}^{2}$, and Dalibor Petrzelka ${ }^{3}$ \\ ${ }^{1}$ Tomas Bata University in Zlin, nam. T. G. Masaryka 5555, 76001 Zlin, Czech Republic \\ ${ }^{2}$ Slovak University of Technology in Bratislava, Institute of Natural and Synthetic Polymers, Vazovova 5, 81243 Bratislava, \\ Slovakia \\ ${ }^{3}$ Siemens, s.r.o. branch of electromotors Frenstat, Markova 952, 74401 Frenstat pod Radhostem, Czech Republic
}

\begin{abstract}
Nowadays, it is very desirable to obtain the low cost polymeric material with the best material properties. For the best modification of the commodity and construction polymeric materials it is firstly necessary to know the basic material properties. In this study the bending and Charpy impact test specimens were fabricated via a professional FDM 3D printer Fortus $900 \mathrm{mc}$, from company Stratasys, processing ABS-M30 in three build orientation XY, XZ-H and XZ-V. The 3D printed test specimens were examined to compare the effect of layer thickness and building orientation. Tensile test machine Zwick 1456 and impact pendulum Zwick HIT50P were used for bending and Charpy impact tests. Optical microscopy was utilized to perform fractography on impact test specimens to explore the effect of the layer thickness and building orientation on the fracture surface morphology of the failed specimens. This study demonstrates the need for material testing for specific processing as additive manufacturing technologies.
\end{abstract}

\section{Introduction}

Nowadays, 3D printing had become more and more used technology for the production of the prototypes, tools and models. There are several commonly used technologies of 3D printing such as selective laser sintering (SLS), selective laser melting (SLM), stereolithography, direct metal laser sintering (DMLS) and the most often used technology fused deposition modelling (FDM) [1]. Another 3D printing technology is PolyJet material jetting process in which the layers of photopolymer are selectively deposited onto a platform via inkjet print heads. The ultra-violet lamps during this process cure the recently deposited photopolymer [2].

FDM is fast growing technology of rapid prototyping using of building complex parts in a reasonable period. Stratasys firstly established FDM technology. Since 2003, there has been the large growth of 3D printers because of price lowering [3]. DMLS and SLS technologies enable to generate 3D parts by solidifying successive layers of material. SLS technology enables to use many various types of materials and has been one of the fastest growing $3 \mathrm{D}$ printing technologies in the last two decades [1].

3D printing is also able to use for the production of specific tools. The advantages in comparison to conventional toolmaking methods are the cost reduction and decrease of delivery time. The better functionality and also the possibility of the adjustment on costumer's demands are other advantages [4]. In our study, The Stratasys Fortus $900 \mathrm{mc} 3 \mathrm{D}$ printer is used. This printer is produced for the production of manufacturing aids and tooling, functional prototypes as well as for short-run digital manufacturing. The large format FDM printer enables the creation with the reliability and the accuracy, using engineering thermoplastics and the rapid installation. This printer is compatible with many materials such as ABS, PC-ABS, ASA, Nylon 6 etc. [5].

A team of scientists investigated thirteen 3D printed samples with the length of $60 \mathrm{~mm}$, the width of $12.5 \mathrm{~mm}$ and thickness of $3.5 \mathrm{~mm}$ using 3D printer Fortus 400 . The blend of PC-ABS was developed by Stratarys Inc combining the mechanical strength of $\mathrm{PC}$ and the flexibility of ABS. They found out that the creep displacement decreases with the decrease of the slice height, air gap and raster fill angle. The air gap of $0 \mathrm{~mm}$, slice height of $0.2540 \mathrm{~mm}$ and raster fill angle of $0{ }^{\circ}$, bead width of $0.4572 \mathrm{~mm}$ and print direction of $20^{\circ}$ were the optimum conditions [6].

A scientific team investigated the 3D printed samples designed using double cantilever beam (DCB) and printed at three various nozzle temperatures. They measured Young's modulus from the filament tensile test. They found out that the fracture resistance exhibited very good repeatability and the adhesion of the interlayer of the samples which were printed at higher temperatures was found to be similar to the fracture resistance of bulk ABS, which has the excellent interlayer bonding [7].

Many research papers have been written about the mechanical behaviour of the polymers. Nowadays the $3 \mathrm{D}$ printing is more spread and because of that it is necessary to test for material properties also $3 \mathrm{D}$ printed

* Corresponding author: pstoklasek@utb.cz

(C) The Authors, published by EDP Sciences. This is an open access article distributed under the terms of the Creative Commons Attribution License 4.0 (http://creativecommons.org/licenses/by/4.0/). 
specimens. Our goal in this study is to compare static and impact bending behaviour of printed parts from ABS with using professional FDM 3D printer Fortus $900 \mathrm{mc}$ in different layer thickness and building orientation. At first, the specimens were printed, then the specimens were subjected to the bending test, Charpy impact test and subsequently to the evaluation of the surface fraction. At the end of this article, the results were discussed and evaluated.

\section{Experimental}

A professional FDM (Fused Deposition Modeling) 3D printer Fortus $900 \mathrm{mc}$ was fed with ABS-M30 filament with a specified diameter of $1.75 \mathrm{~mm}$ supplied by Stratysys company. Five specimens were printed at three orientation layer direction (XY, XZ-H and XZ-V), as it is described in Figure 1, for each test method. Bending test and Charpy impact test with and without notch were used for testing prepared specimens.

3 -point bending test was conducted in compliance to ISO 178 using a Zwick 1456 universal tensile testing machine equipped with a $5 \mathrm{kN}$ load cell at a crosshead speed of $50 \mathrm{~mm} / \mathrm{min}$.

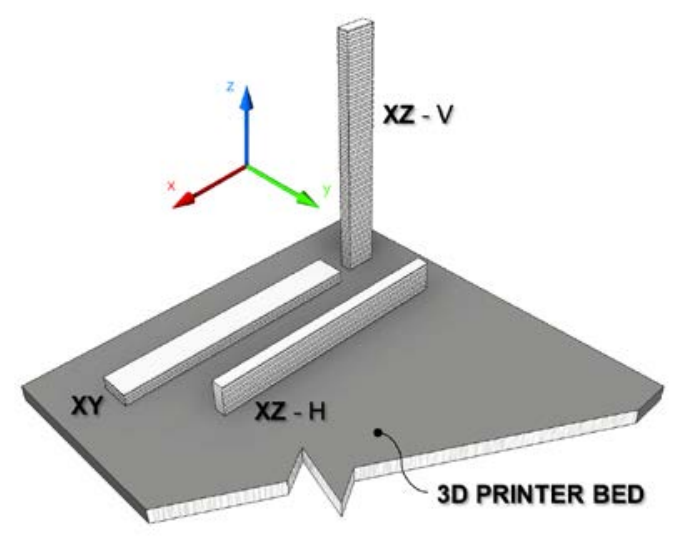

Fig. 1. Building orientation of 3D printed specimens

The Charpy impact test was carried out on Zwick HIT50P equipment at an ambient temperature of $23{ }^{\circ} \mathrm{C}$ according to the ISO 179-2 standard. In this impact hammer test, $50 \mathrm{~J}$ of potential energy was used for research purposes. 5 samples were tested at each orientation and layer (Figure 2), and their maximum impact force and impact strength values with or without notched samples (Figure 3) were evaluated in the TestXpert II, MS Excel and MiniTab 16 programmes. Arithmetic mean and standard deviation were used as the statistical parameters in this measurement process. Conditioning for all specimens was taken for 5 days in temperature of $23{ }^{\circ} \mathrm{C}$ and $50 \%$ of relative humidity.

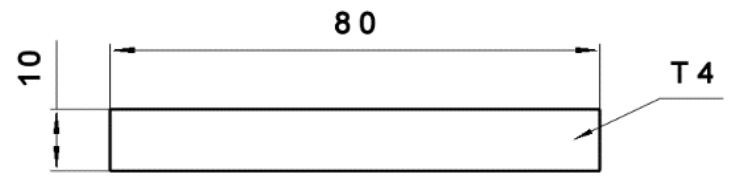

Fig. 2. Dimensions of testing specimen type 1 for bending and Charpy impact test, notched one has notch with height $2 \mathrm{~mm}$ in the middle of specimen from one side.

\section{Results and discussion}

In Figure 4 is depicted two layers at $0.17 \mathrm{~mm}$. From optical measurement on microscope Zeiss Axio Scope A1 was found that the real value of layer thickness is $0.178 \mathrm{~mm}$.

\subsection{Bending test}

The first 3-point bending test was used at crosshead speed $50 \mathrm{~mm}$ per minute. From the measurements flexural modulus, flexural strength and deflection at break were evaluated and depicted in the figures where is compared printed parts at different layers and building orientations.

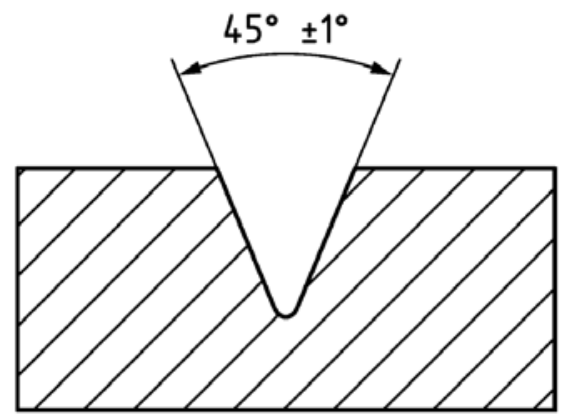

Fig. 3. Dimensions of notch type $\mathrm{C}$ for Charpy impact test, radius of tool tip $0.1 \mathrm{~mm} \pm 0.02 \mathrm{~mm}$ was used.

Graphical comparison of flexural modulus at the different layer and orientation is depicted in Figure 5. In this figure arithmetic mean and standard deviation as a main statistical parameters are used. The highest flexural modulus was measured at orientation XZ-H at each layer thickness, the value is about of $2100 \mathrm{MPa}$.



Fig. 4. ABS layer measurement for printed layer $0.17 \mathrm{~mm}$. 


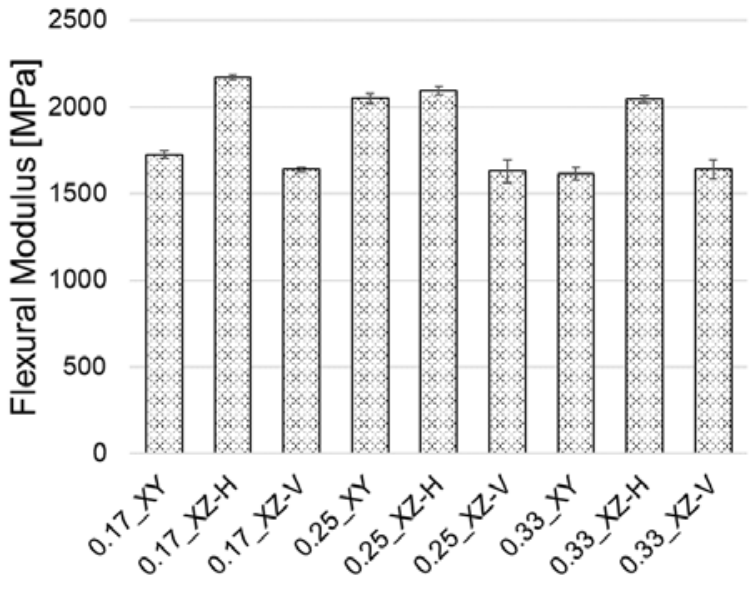

Fig. 5. Flexural modulus of $3 \mathrm{D}$ printed parts.

In Figure 6 is shown comparison of flexural strength in dependence on layer thickness and orientation. Layer thickness has just small influence to flexural strength; however, building orientation, especially at direction $\mathrm{XZ}-\mathrm{Z}$ has huge impact to decreasing flexural strength.

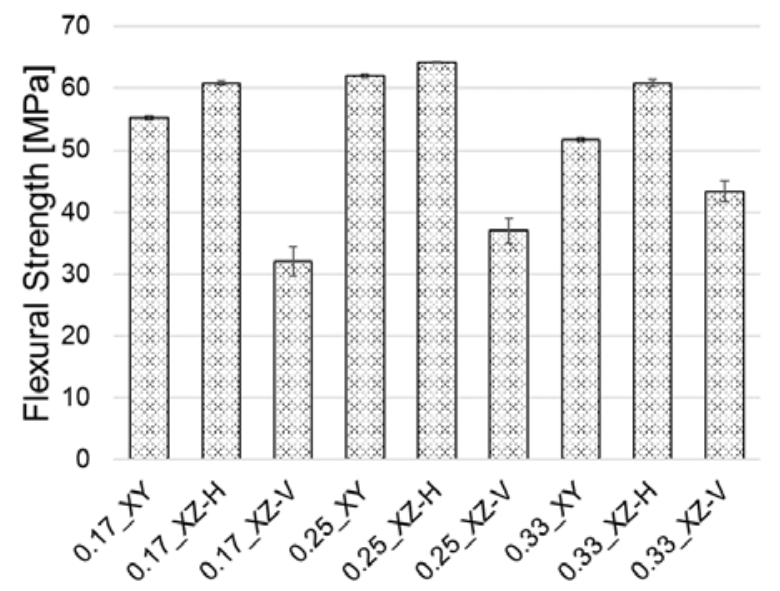

Fig. 6. Flexural strength of 3D printed parts.

In Figure 7 is depicted comparison of deflection at break at different layer thickness and build orientation. It is clear from this measurement that deflection at break is rising with increasing layer thickness at every orientation. The lowest values were measured at building orientation $\mathrm{XZ}-\mathrm{V}$.

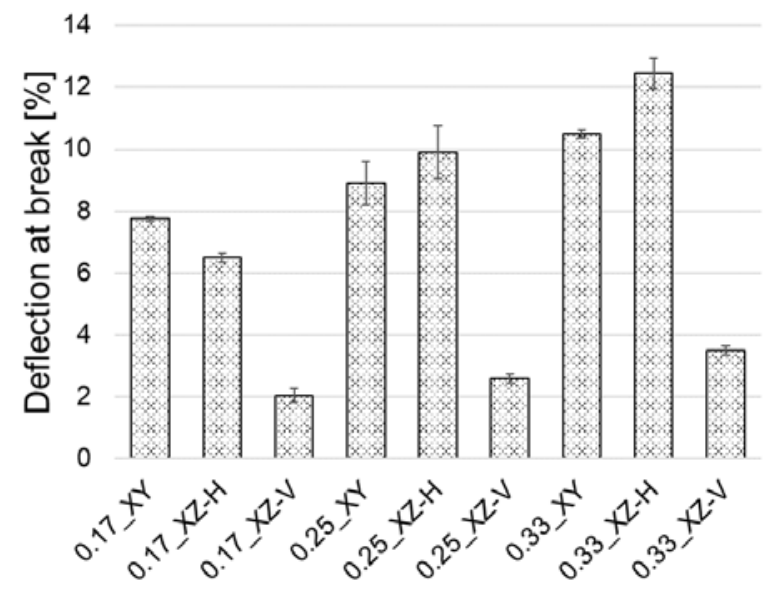

\subsection{Charpy impact test}

The second charpy impact test was used at potential pendulum energy $50 \mathrm{~J}$. From the measurements maximum impact force, impact strength and notched impact strength were evaluated and depicted in the figures where is compared printed parts at different layers and building orientations.

In Figure 8 maximum impact force comparison without notch is depicted. The layer thickness in this measurement do not have influence to maximum impact force. Every measurement is in error bars. On the other hand, building orientation can cause huge decrease of maximum impact force, especially at XZ-V orientation, where decrease is about of $33 \%$ in comparison with XZ$\mathrm{H}$ orientation



Fig. 8. Maximum impact force at Charpy impact test.

In Figure 9 maximum impact force comparison with notch is shown. The layer thickness in this measurement plays important role. Layer thickness 0.17 at building orientation $\mathrm{XY}$ and $\mathrm{XZ}-\mathrm{H}$ is double times higher than other higher layer thickness at the same building orientation. On the other hand, building orientation XZ$\mathrm{V}$ causes at every layer thickness rapid decrease and the lowest value of maximum impact force at notched specimens.

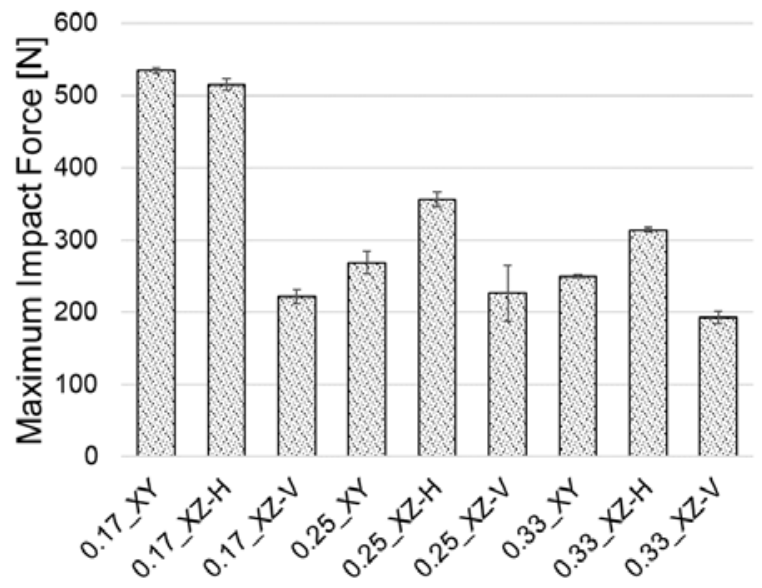

Fig. 9. Maximum impact force at Charpy notched impact test.

Fig. 7. Deflection at break of 3D printed parts. 


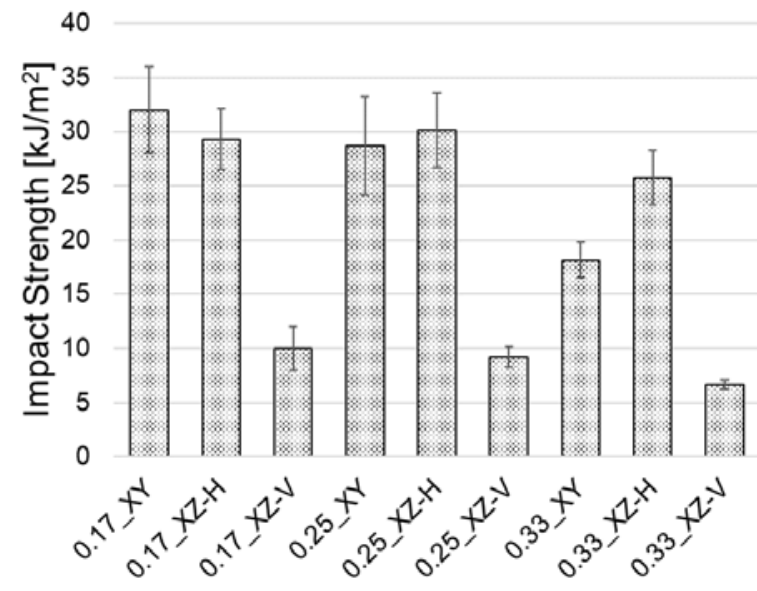

Fig. 10. Impact strength of 3D printed parts.

In Figures 10 and 11 impact strength and notched impact strength can be seen, respectively. The trend is similar with Figures 8 and 9. It follows from this measurement that notch has huge influence on bending behaviour (both static and impact).



Fig. 11. Notched impact strength of 3D printed parts.

\subsection{Fracture surface evaluation}

In Figures 12, 13 and 14 are shown fracture surfaces of ABS specimens at layer thickness $0.17 \mathrm{~mm}$ after the bending test. It can be seen from Figure 12 that the crack spread along oriented printed structure $\left(45^{\circ}\right)$. From Figure 13 is possible to see delamination of lower layer which was tensile loaded. The last Figure $14 \mathrm{XZ}-\mathrm{V}$ orientation fracture surface is depicted. Here is separated layer from layer, where the lowest toughness of the whole system was observed.

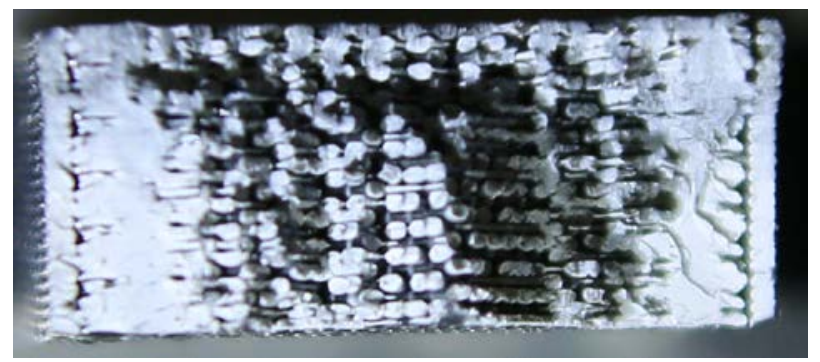

Fig. 12. ABS fracture surface at $X Y$ orientation $(0.17 \mathrm{~mm}$ printed layer), after bending test.

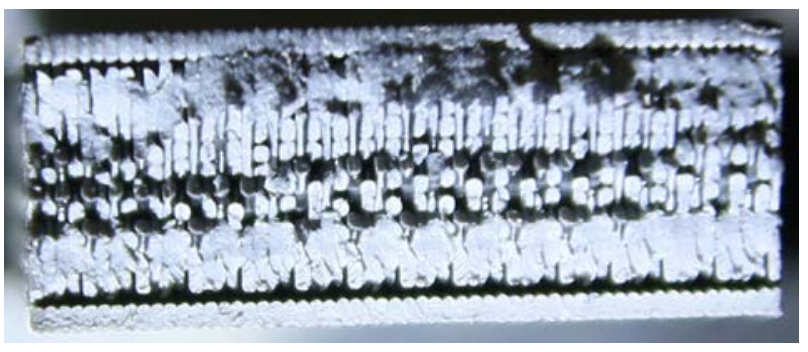

Fig. 13. ABS fracture surface at $\mathrm{XZ}-\mathrm{H}$ orientation $(0.17 \mathrm{~mm}$ printed layer), after bending test.



Fig. 14. $A B S$ fracture surface at $X Z-V$ orientation $(0.17 \mathrm{~mm}$ printed layer), after bending test.

\section{Summary}

The presented study shows a case for the establishment of bend testing (static and impact) standards specifically for 3D-printed specimens. Bending test and Charpy impact test (with and without notch) of specimens printed from ABS-M30 on a professional FDM 3D printer Fortus $900 \mathrm{mc}$ from company Stratasys yielded results which were statistically evaluated at each layer thickness and building orientation. From this measurement implies that layer thickness can have influence on bending behaviour, especially when there is some notch or fast change of wall thickness. On the other hand, the huge influence has building orientation of printed parts. The lowest value of flexural and impact strength was measured at orientation XZ-V. Because 3D printing is every moment more spread, it is desirable to compare material properties of 3D printed specimens in more axes and subsequently to compare to commonly produced specimens (e.g. injection moulded) and because the common material properties of commonly processed $\mathrm{ABS}$ are already known, 3D printed specimens are needed to study in the depth to obtain the deeper knowledge about this material processed using more technologies.

This paper is supported by the internal grant of TBU in Zlin No. IGA/FT/2018/012 funded from the resources of specific university research, by the Czech Ministry of Industry and Trade in Program "Aplikace" No. CZ 01.1.02/0.0/0.0/16_084/0009949 and by the Ministry of Education, Youth and Sports of the Czech Republic within the National Sustainability Programme project No. LO1303 (MSMT-7778/2014) and also by the European Regional 
Development Fund under the project CEBIA-Tech No. CZ.1.05/2.1.00/03.0089.

\section{References}

1. H. Mazhar, T. Osswald, D. Negrut, Additive Manufacturing 12 291-295 (2016)

2. J.P. Moore, CH.B. Williams, Rapid Prototyping J. 21 675-685 (2015)

3. M. Jurcisin, B. Marek, S. Jan, Appl. Mech. Matter. 827 69-72 (2016)

4. P. Zeleny, T. Vana, J. Stryal, Mater. Sci. Forum 862 316-323 (2016)

5. Mazak Corp., Modern Equipment Review, mmsonline.com, August 151-152 (2017)

6. O.A. Mohamed, S.H. Masood, J.L. Bhowmik, Mater. Lett. 193 58-62 (2017)

7. N. Aliheidari, R. Tripuraneni, A. Ameli, S. Nadimpalli, Polym. Test. 60 94-101 (2017) 